\title{
Männliche Tatkraft und weibliches Sein: eine Radioidylle 1934-1940
}

MONIKa PATER

Kurz nach der ersten deutschlandweiten Jubelfeier zum Erntedankfest strahlte der Deutschlandsender am 3. Oktober 1933 die erste Folge der idyllischen Sendereihe »Deutscher Kalender: Ein Monatsbild vom Königswusterhäuser Landboten« aus. In den leitmotivischen Schlussversen der insgesamt 90 Folgen ${ }^{1}$ dieser Reihe wird deutlich, dass über die immer wiederkehrende Thematisierung der Gegensätzlichkeiten von Stadt und Land eine Aufwertung des Landlebens stattfinden sollte, denn am Ende jeder Folge hieß es:

»Du zarter Städter, spotte nicht

der schwielenvollen Hand,

sie nähret, was dein Stolz auch spricht,

dich und das ganze Land. $\ll^{2}$

1 I Ausgestrahlt von Oktober 1933 bis Mai 1940 brachte es die Sendereihe zusammen mit Sondersendungen zu Festtagen und Sendejubiläen auf 90 Folgen; davon sind im Nachlass Raschke in der Sächsischen Landesbibliothek/Staats- und Universitätsbibliothek Dresden (im Folgenden SLUB Dresden) 58 Manuskripte erhalten, vgl. Hans-Ulrich Wagner: »Kommentierte Radiographie«, in: Ders., Günter Eich und der Rundfunk. Essay und Dokumentation, Potsdam: Verlag für Berlin-Brandenburg 1999, S. 107-376, hier S. 122. Bei dem Folgenden handelt es sich um eine stark überarbeitete Fassung des Kapitels »Völkische Unterhaltung: die Monatsbilder des Königswusterhäuser Landboten«, in: Monika Pater: »Rundfunkangebote«, in: Marßolek/Saldern (Hg.), Zuhören und Gehörtwerden 1 (1998), S. 172-187.

2 | Zitiert nach Hans-Ulrich Wagner: »Essay«, in: Ders., Günter Eich und der Rundfunk, S. 25-106, hier S. 51. 
Die Monatsbilder des »Königswusterhäuser Landboten« wurden nach und nach zu einem Aushängeschild des NS-Rundfunks. ${ }^{3}$ Mit zunehmendem Erfolg wurde die Sendereihe immer stärker Bestandteil der Propagierung der NS-Volksgemeinschaft im Radio. 〉Erfolg〈 im Sinne von Hörerzahlen lässt sich rückblickend nur an Indizien festmachen: $\mathrm{Zu}$ diesen gehören die lange Sendedauer von sieben Jahren, die Ausstrahlung von Sondersendungen zu Feiertagen wie Ostern und Weihnachten und zur Rundfunkausstellung sowie die Beschreibung der Reaktionen der Hörer/-innen in den Pressemitteilungen des Deutschlandsenders. ${ }^{4}$ Auch die zunehmende Inanspruchnahme für die NS-Propaganda kann so gelesen werden, z. B. wurde 1937 in einzelnen Folgen der Sendung für »Kraft durch Freude« ebenso geworben wie für den freiwilligen Einsatz als Erntehelfer. ${ }^{5}$ Für den Erfolg dieses Konzeptes spricht auch die Existenz einer Nachahmersendung »Der märkische Kalendermann sagt den neuen Monat an ${ }^{6}{ }^{6}$

Im Folgenden interessiert, welche gesellschaftliche Ordnung in ausgewählten Folgen der Sendereihe (re)präsentiert und wie diese von der Kategorie Geschlecht strukturiert wurde. ${ }^{7}$ Bei den Repräsentationen von Weiblichkeit und Männlichkeit handelt es sich um gesamtgesellschaftliche Konstruktionen, »die die spezifischen kulturellen, gesellschaftlichen, historischen und psychologischen Bedingungen reflektieren, die sie hervorbringen ${ }^{8}{ }^{8}$ Dies ist keine Einbahnstraße: Gesellschaftliche Rahmenbedingungen, die innerhalb medialer Erzählungen mit ihren spezifischen Vorstellungen von Weiblichkeit und Männlichkeit entstehen, prägen zwar

3 | Vgl. Glenn R. Cuomo: Career at the Cost of Compromise. Günter Eich's Life and Work in the Years 1933-1945, Amsterdam u. a.: Rodopi 1989, S. 80.

4 | Vgl. M. Pater: »Rundfunkangebote«, a. a. O., S. 172 ff.

5 | Vgl. G. R. Cuomo: Career; Axel Vieregg: Der eigenen Fehlbarkeit begegnet. Günter Eichs Realitäten 1933-1945, Eggingen: Edition Isele 1993; H.-U. Wagner: »Radiographie«, a. a. O., S. 183.

6 | Diese von Günter Eich für den Berliner Rundfunk geschriebene Sendereihe mit 24 monatlichen Folgen lief von August 1937 bis Juli 1939, vgl. H.-U Wagner: »Radiographie«, a. a. O., S. 178.

7 | Die Sendereihe bietet sich aus mehreren Gründen für eine solche Analyse an: wegen der Dauer der Ausstrahlung und der überlieferten Sendemanuskripte, die eine Entwicklung über einen längeren Zeitraum deutlich machen (Manuskripte anderer fiktionaler Serien sind m. E. nicht erhalten). Zudem kann der Beitrag auf Forschungen aufbauen, die sich der Autorenschaft des in der BRD bekannten Schriftstellers Günter Eich verdanken und die den Entstehungskontext und das Umfeld u. a. dieser Serie erarbeitet haben. Vgl. G. R. Cuomo: Career; H.-U. Wagner: Günter Eich und der Rundfunk; Wilhelm Haefs/Walter Schmitz (Hg.): Martin Raschke (1905-1943): Leben und Werk, Bamberg: Thelem 2002; Hans-Ulrich Wagner: ")Der Weg in ein sinnhaftes, volkhaftes Leben.« Die Rundfunkarbeiten von Martin Raschke«, in: Wilhelm Haefs/Walter Schmitz (Hg.), Martin Raschke (1905-1943): Leben und Werk, Bamberg: Thelem 2002, S. 79-106; Hans-Ulrich Wagner: »Eine Karriere ohne Kompromiss. Martin Raschke und der Rundfunk 1928-1940«, in: Rundfunk und Geschichte 28 (2002), Heft 1/2, S. 5-21.

8 | Gitta Mühlen-Achs/Bernd Schorb: »Einleitung«, in: Dies. (Hg.), Geschlecht und Medien, München: KoPäd Verlag 1995, S. 7-12, hier S. 10. 
die Vorstellungen von Geschlecht. Jedoch besitzen narrative Texte gleichzeitig eine »performative Qualität«, d. h., Erzählungen bringen Vorstellungen von Weiblichkeit und Männlichkeit ihrerseits »aktiv hervor, indem sie sie mit narrativen Mitteln inszenieren «. ${ }^{9}$ Je stärker Medien die Gesellschaft durchdringen, umso bedeutender ist ihr Stellenwert bei der Herstellung dieser Konstruktionen. ${ }^{10}$

Als Analysegegenstand beinhalten die `Relikte〈 populärer Unterhaltungssendungen - ähnlich wie Texte beispielsweise literarischer Provenienz - ein Problem: Das Verhältnis zwischen dem Text und den Rezipientinnen und Rezipienten. Jeder Text weist sprachliche und strukturelle Merkmale auf, die den Spielraum für die Interpretation abstecken. $\mathrm{Zu}$ den verschiedenen Möglichkeiten zählt hierbei auch die von den Herrschenden erwünschte Interpretation, eine Interpretation, die mit den grundlegenden Werten der Gesellschaft übereinstimmt und zur Stabilisierung gesellschaftlicher Verhältnisse beiträgt. Welche Lesart tatsächlich hergestellt wird, hängt dabei sowohl vom Text ab, dessen Struktur und Art die Zuweisung von Sinn durch die Rezipientinnen und Rezipienten vorstrukturiert, als auch von den rezipierenden Personen, deren Einstellungen und soziale Position die Akzeptanz spezifischer Lesarten bestimmen. Die jeweilig aktualisierte bzw. hergestellte Bedeutung wird daher weder allein durch den Text vorgegeben noch allein von den Rezipientinnen und Rezipienten festgelegt.

Eine Unterhaltungssendung wie der »Königswusterhäuser Landbote«, für ein breites Publikum zu einer guten Sendezeit konzipiert, verstand sich vermeintlich politikfern als Entlastungs- und Entspannungsangebot - ein doppelter Grund hinzuschauen, wie dieses Angebot als Vermittlungsinstanz gesellschaftlicher Ordnungsvorstellungen funktioniert hat. Die fiktionalen, unterhaltend intendierten Texte werden auf den folgenden Ebenen in Hinblick auf Entwürfe von Weiblichkeit und Männlichkeit analysiert: der narrativen Struktur (Wer spricht bzw. wie wird erzählt?) und derjenigen des erzählten Geschehens (Plot) bzw. der erzählten Figuren (Worüber/Über wen wird gesprochen?). Eine zentrale Vorentscheidung jedes Autors bzw. jeder Autorin ist die Festlegung der Art des Textes, d. h. der Gattung, denn die Bandbreite der möglichen Entwürfe von Weiblichkeit und Männlichkeit wird davon bestimmt. Daher müssen »die gattungsspezifischen Möglichkeiten narrativer Inszenierung von [...] Geschlecht « reflektiert werden. ${ }^{11}$ Zunächst werden daher Gattung und Entstehungskontext der Sendereihe, dann die Entwürfe von Geschlecht auf der Handlungsebene betrachtet, um zuletzt auf die Erzählstruktur einzugehen.

9 | Ansgar Nünning/Vera Nünning: »Making Gendered Selves: Analysekategorien und Forschungsperspektiven einer gender-orientierten Erzähltheorie und Erzähltextanalyse«, in: Nieberle/Strowick (Hg.), Narration und Geschlecht (2006), S. 23-44, hier S. 26.

$10 \mid$ Zur Verbreitung des Radios und seiner Verankerung im Alltag der 1930er Jahre vgl. Uta Schmidt: »Radioaneignung«, in: Marßolek/Saldern (Hg.), Zuhören und Gehörtwerden 1 (1998), S. 243-360.

11 | Nadyne Stritzke: »(Subversive) Narrative Performativität. Die Inszenierung von Geschlecht und Geschlechtsidentitäten aus Sicht einer gender-orientierten Narratologie«, in: Nieberle/Strowick (Hg.), Narration und Geschlecht (2006), S. 93-116, hier S. 93. 
Gegenstand der Analyse sind Sendemanuskripte und gedruckte Auszüge aus der Sendung, die das flüchtige Hörerlebnis nachlesbar machen, die materiellen Überbleibsel eines komplexen Kommunikationsprozesses. Dieser relationale Prozess entsteht zwischen den Produzenten der Sendung und den Hörenden in einer spezifischen sozialen Situation. Teil dieses Prozesses ist Sekundärmaterial wie Besprechungen und Auszüge aus Programmzeitschriften, ${ }^{12}$ das einen Rahmen für die Interpretation der Hörenden setzt und die von den Produzenten erwünschte Interpretation wiedergibt. Der Kommunikationsprozess als Ganzes ist nicht mehr zu rekonstruieren, aber man kann versuchen die Überbleibsel zu interpretieren und plausible Schlussfolgerungen über Nutzung und Akzeptanz zu ziehen. ${ }^{13}$ Ausgangspunkt für solche Schlussfolgerungen ist, dass der Umgang mit medialen Repräsentationen aller Art situativ im Alltag der Rezipientinnen und Rezipienten verankert ist und auf ihre Lebenswelt Bezug nimmt.

Keine Folge der Sendereihe wurde aufgezeichnet und überliefert. ${ }^{14}$ Daher liegt mit den Manuskripten nur so etwas wie eine >halbe Quelle` vor. Die akustische Umsetzung hat die Erzählweise unterstützt und zur Bedeutungszuweisung der Hörenden beigetragen.

\section{Entstehung und gattungsbedingte Merkmale der Sendereihe}

Der Name der Sendereihe $»$ Deutscher Kalender: Monatsbilder vom Königswusterhäuser Landboten« war programmatisch:

- Die Begriffe Kalender und Monatsbild verweisen auf die literarische Form der Kalendergeschichte mit ihrer moralischen und belehrenden Absicht, ebenso wie Bote, denn Kennzeichen der Gattung war die Personifizierung des Kalenders in einem >Kalendermann , der sich als Dialogpartner des Lesers anbot. ${ }^{15}$

- Deutsch verweist auf die Sagen und Legenden aus dem deutschen Sprachraum sowie auf (hier dörfliches) Leben in Deutschland. Durch den Ausschluss dessen,

12 | Erhalten sind Ankündigungen und Besprechungen im Deutschen Rundfunkarchiv Frankfurt/Main (im Folgenden DRA), Pressemitteilungen DS 1933-1936, passim, BA Koblenz, R 78, Nr. 830 und 831, passim. Auch H.-U. Wagner: »Radiographie«, a. a. O., zitiert sie zu einzelnen Sendungen. Zur Diskussion über Stellenwert und Aussagekraft von Sekundärmaterial vgl. M. Pater: »Rundfunkangebote«, a. a. O., S. 134.

13 | Vgl. Klaus Merten: Inhaltsanalyse. Eine Einführung in Theorie, Methode und Praxis, Opladen: Westdeutscher Verlag 1980, S. 57.

14 | Aufzeichnen war technisch und organisatorisch aufwändig, man konzentrierte sich auf `wichtige«, vor allem politische Ausstrahlungen, vgl. M. Pater: »Rundfunkangebote«, a. a. O., S. $140 \mathrm{f}$.

15 | Jan Knopf: »Kalendergeschichte«, in: Harald Fricke (Hg.), Reallexikon der deutschen Literaturwissenschaft. Neubearbeitung des Reallexikons der deutschen Literaturgeschichte, 3. Aufl., Berlin, New York: W. de Gruyter 2000, S. 217 f. 
was als »nichtdeutsch« betrachtet wird, verweist dieses Adjektiv auch auf die »arische Volksgemeinschaft«.

- Königs Wusterhausen nahe Berlin ist der Standort des Sendemastes. Von hier wurde die erste Rundfunksendung ausgestrahlt. Gleichzeitig ist Königs Wusterhausen der Standort des Landboten, hier hat er in der Erzählung >seine Kammer`, von hier aus zieht er aufs Land.

Die Figur des Landboten fungiert in mehrfacher Weise als narratives Bindeglied: Da er beim Rundfunk angestellt ist und von Königs Wusterhausen aus in unterschiedliche Landstriche aufbricht, verbindet er Stadt und Land, Moderne (hierfür steht der Rundfunk) und Tradition. In den Folgen wird nicht gesagt, welche Botschaften der Landbote in die dörflichen Gemeinschaften bringt, so dass die Funktion dieses Charakters innerhalb des dargestellten sozialen Gefüges nicht eindeutig ist. Wie gezeigt werden wird, bestand die Botschaft des Landboten im Angebot von Ordnungsvorstellungen, die Halt und Orientierung gaben. So versprachen die Folgen des Jahres 1935 den Hörenden Geborgenheit:

»Die Sendung [...] lauscht dem Ablauf des Jahres dort, wo es sich in ewigem Gleichmaß vollendet. Von Regen und gut Wetter ist die Rede. Dazwischen erklingen alte Volksweisen. Unser Landbote fühlt sich daheim, er preist den Segen der Erde. [...] Es ist ein ruhvolles Bild, ein sinniges Bedenken in der Jagd der Zeit. $\ll^{16}$

Dieses Zitat fasst den Charakter der Sendereihe zusammen: Im Jahresverlauf wurden Geschichten erzählt - die Sendung bezog sich explizit wie implizit auf den Kalender als Ordnungsprinzip und entsprach so in jeder Hinsicht der Definition einer Kalendergeschichte. ${ }^{17}$ Als Vorbild der Kalendergeschichten des 19. Jahrhunderts galt »Das Schatzkästlein des Rheinischen Hausfreundes« (1811) von Johann Peter Hebel; die dort entwickelten Kennzeichen - »volkstümliche Elemente [wie] landschaftliche Gebundenheit, bäuerliches und kleinbürgerliches Personal « ${ }^{18}$ - prägten auch die Monatsbilder des »Königswusterhäuser Landboten«. Da Günter Eich, einer der Autoren des »Königswusterhäuser Landboten«, 1933 Hebels »Schatzkästlein« für den Rundfunk bearbeitet hatte, ${ }^{19}$ war er mit dieser im 19. Jahrhundert sehr popu-

16 | »Hör mit mir«, Nr. 31, 28.7.1935, S. 9, zitiert nach H.-U. Wagner: »Radiographie«, a. a. O., S. 152 .

17 | Vgl. J. Knopf: »Kalendergeschichte«, a. a. O., S. 217.

18 | Vgl. ebd., S. 219.

19 | Vgl. H.-U. Wagner: »Essay«, a. a. O., S. 48. Die Verarbeitung von Ausschnitten aus dem »Schatzkästlein« in der Folge vom 1. November 1935 zeigt zudem dessen Nähe zu der Sendereihe; diese hieß ab dieser Folge bis Dezember 1936 »Aus der Truhe des Königswusterhäuser Landboten«. Durch die Konzeptänderung wurde die fiktionale Handlung auf ein Minimum reduziert, um mehr Lieder und Gedichte einbauen zu können. Vgl. H.-U. Wagner: »Radiographie«, a. a. O.; siehe auch H.-U. Wagner: »Essay«, a. a. O.; die Rückkehr zu den Monatsbildern mit einer stärker ausgearbeiteten fiktionalen Handlung ab Januar 1937 deutet darauf hin, dass diese Änderung den Hörenden eher nicht gefiel. 
lären Form vertraut, bevor die Sendereihe konzipiert wurde. In den Monatsbildern wurde neben einer fiktionalen, oft nur losen Handlung aus dem dörflichen Geschehen das Brauchtum, das mit dem jeweiligen Monat verbunden war, geschildert: beispielsweise das Dreikönigsschießen im Januar, das Aufrichten eines Maibaums und die Wahl der Maienkönigin oder die Martinsgans im November. Damit präsentierte die Sendereihe »die kostbarsten Schätze deutschen Volkstums«, so der Pressedienst des Deutschlandsenders im Rückblick 1937. ${ }^{20}$

Hans-Ulrich Wagner hat herausgearbeitet, dass die Idee zum »Königswusterhäuser Landboten« »einem breiten publizistischen Diskurs« Ende der 1920er/Anfang der 1930er Jahre entstammte, der den »Gegensatz zwischen Stadt und Land, Metropole und Provinz« herausstellte. ${ }^{21}$ Im Kontext des ersten feierlich-propagandistischen Begehens des Erntedankfestes am 1. Oktober 1933 entwickelte Günter Eich zusammen mit dem Leiter der literarischen Abteilung des Deutschlandfunks, Werner Pleister, das Grundkonzept der Sendereihe. ${ }^{22}$ Bis 1940 schrieben die jungen, national-konservativen Schriftsteller Günter Eich und Martin Raschke abwechselnd, oft auch gemeinsam, die monatlichen Hörfolgen. In den 1920er Jahren gehörten die beiden »jener Gruppe von Schriftstellern an, die sich insgesamt apolitisch und antifortschrittlich gibt, die den im Expressionismus artikulierten Hass gegen die Großstadt tradiert, die sich antizivilisatorisch zurückzieht und Naturgedichte schreibt $\ll .{ }^{23}$ Hier werden die Anknüpfungspunkte zu Versatzstücken der NS-Ideologie deutlich, die es Eich und Raschke erleichterten, während des politischen Umbruchs im Jahr 1933 im Medium Rundfunk erfolgreich und dauerhaft Fuß zu fassen. In Bezug auf Raschke spricht Wagner sogar von einer »mühelosen [Transformation] in die >Volksgemeinschafts<-Ideologie«, die am deutlichsten in den Kalenderbildern des »Königswusterhäuser Landboten« zutage trete. ${ }^{24}$

Die Sendereihe wurde in der ersten Phase nationalsozialistischer Programmgestaltung konzipiert, in der »energisch politisiert« wurde. ${ }^{25}$ Der Rundfunk galt führenden Nationalsozialisten als »Vortrupp nationalsozialistischer Weltanschauung«, er sollte großen Anteil daran haben, die Zustimmung einer Mehrheit der Deutschen zum NS-Regime zu erreichen ${ }^{26}$ und sie auf die $»$ Volksgemeinschaft« einzuschwö-

$20 \mid$ BA Koblenz, R 78, Nr. 831, Pressedienst Deutschlandsender (Oktober 1937).

21 | Vgl. H.-U. Wagner: »Essay«, a. a. O., S. 49 f.

22 | Vgl. ebd. Hier stellt Wagner - vermutlich von zeitgenössischer Rezeption beeinflusst (vgl. H.-U. Wagner: »Radiographie«, a. a. O., S. 183) - die Kalenderbilder in die Tradition der Handels- und Volkszeitung »Wandsbeker Bothe«.

23 | H.-U. Wagner: »Essay«, a. a. O., S. 29.

$24 \mid$ H.-U. Wagner: »Karriere ohne Kompromiss«, a. a. O., S. 14.

25 | Vgl. Reichsminister Goebbels vor den Rundfunk-Intendanten, in: Mitt. RRG, Nr. 409 vom 11.4.1934, Bl. 1-2, hier Bl. 1; ausführlicher zu den einzelnen Phasen der NS-Programmgestaltung: Daniela Münkel: »Die Entwickung der Programmstruktur«, in: Marßolek/Saldern (Hg.), Zuhören und Gehörtwerden 1 (1998), S. 95-105.

26 | Eugen Hadamovsky: Der Rundfunk im Dienste der Volksführung (Gestalten und Erscheinungen der politischen Publizistik, 1), Leipzig: Noske 1934, S. 20. 
ren. ${ }^{27}$ Mit der Betonung einfacher Ländlichkeit erwies sich die Sendereihe als gute Möglichkeit, nationalsozialistische Ideologie vergleichsweise unaufdringlich $\mathrm{zu}$ verbreiten. Sie eignete sich vor allem dazu, die Bildung der »Volksgemeinschaft $«$ aus verschiedenen »Stämmen« und unter Überwindung des Stadt-Land-Gegensatzes darzustellen. ${ }^{28}$

Besonders deutlich wird das an der zusätzlichen Sendung »Weihnachtsreise des Königswusterhäuser Landboten« am 25. Dezember 1934. ${ }^{29}$ Aus Anlass der »deutschen « Weihnacht wurde hier die »Volksgemeinschaft« inszeniert und gefeiert: Auf einer Deutschlandreise `besucht〈 der Landbote »alle deutschen Stämme«. Jeder der angeschlossenen regionalen Reichssender präsentiert regionale Weihnachtsmusik sowie etwas Typisches aus seinem Sendegebiet, wobei das Spektrum von einer Begegnung mit Sagenfiguren über Weihnachtslieder von Bach, das Glockengeläut des Kölner Doms bis hin zu Weihnachtsbräuchen reicht. ${ }^{30}$ Die Zusammengehörigkeit aller, die in diesen Regionen wohnen und angeblich unterschiedlichen Sitten und Gebräuchen nachgehen, wurde durch die Figur des aus der Hauptstadt kommenden Landboten versinnbildlicht: Zum Schluss »läßt [der Landbote in seinem Zimmer] noch einmal beim Klang der Glocken der verschiedenen Landschaften alle Erlebnisse an seinem Auge vorüberziehen, die ihm zeigten, in wie vielfältigen Formen überall auf deutsche Art Weihnachten gefeiert wird «. ${ }^{31}$

Vieregg charakterisiert die Hörfolgen des »Königswusterhäuser Landboten« als »umfangreichste und weitreichendste Idyllenproduktion der NS-Zeit, [die] letztlich zur Entmündigung und Entbindung des Untertanen von politischer Verantwortung« beitrug. ${ }^{32}$ Als idyllisch werden Texte bezeichnet, die »einfach-friedliche, meist ländliche Lebensformen als Korrektiv zur Wirklichkeit« schildern; »Motiv-

27 | Vgl. H.-U. Wagner: »Essay«, a. a. O. Eine Analyse der Art und Weise, wie in der Sendung die »Volksgemeinschaft« inszeniert wurde, findet sich in M. Pater: »Rundfunkangebote«, a. a. O., S. $172-187$.

28 | Vgl. Vieregg: Fehlbarkeit, S. 27.

29 | Alle Sender wurden von 18.00 bis $20.00 \mathrm{Uhr}$ zu »einer großen Ringsendung« zusammengeschlossen, vom Deutschlandsender wurde die Sendung koordiniert, vgl. DRA Frankfurt/ Main, Pressedienst RS Berlin (Dezember 1934), S. 1-3.

30 | Vgl. SLUB Dresden, App. 2531, Ms.-Nr. 2072; in Ostpreußen begegnet der Königswusterhäuser Landbote dem Schimmelreiter, in Bayern wohnt er dem Weihnachtsschießen in Berchtesgaden bei, auf dem Berliner Weihnachtsmarkt unterhalten sich der »Urberliner Nante« und ein von einer Weltreise zurückgekehrter, ehemaliger Berliner über vergangene und zukünftige Weihnachtsfeste etc.; die Betonung der Unterschiedlichkeit der »Stämme« und ihrer Zusammengehörigkeit erfolgt über die Pressemitteilungen, siehe DRA Frankfurt/Main, Pressedienst RS Berlin (Dezember 1934).

31 I DRA Frankfurt/Main, Pressedienst RS Berlin (Dezember 1934), S. 4.

32 | A. Vieregg: Fehlbarkeit, S. 27; siehe auch Wolfram Wessels: Hörspiele im Dritten Reich. Zur Institutionen-, Theorie- und Literaturgeschichte, Bonn: Bouvier 1985, S. 434 f.; zur Auseinandersetzung über Eichs Rolle während des Nationalsozialismus vgl. Holger A. Pausch/ Marianne Herzog: »Vergessene Texte, Schrift und Sprache. Beobachtungen zur Günter-EichKontroverse«, in: Wirkendes Wort 45 (1995), Heft 1, S. 133-150. 
und Strukturelemente der Idylle« gehen hier in die Form der Kalendergeschichte ein. ${ }^{33}$ Wie sich im »Königswusterhäuser Landboten« der Bezug auf zeitgenössische Erfahrungen - kennzeichnend für die Kalendergeschichte - mit der Konstruktion idyllischer Orte verband und dies grundlegend durch die Kategorie Geschlecht strukturiert wurde, soll im Folgenden gezeigt werden.

\section{Männliche Tatkraft}

Spätestens durch die Studie von Detlev Peukert ${ }^{34}$ wurde deutlich, dass die rassistisch definierte NS-Volksgemeinschaft in hohem Maße eine Leistungsgemeinschaft war. Leistungen waren an der Stelle zu erbringen, an die das Leben oder das Schicksal einen gestellt hatte. Dazu thematisierte die Sendereihe zeitgenössisch vorhandene Möglichkeiten: So kehrt in der Dezembersendung 1937 ein Charakter, Thomas, aus den USA in sein Dorf im Erzgebirge zurück. ${ }^{35}$ Zwischen ihm und seinem zuhause gebliebenen Jugendfreund, dem Berger-Karl, wird in der Folge ein Kontrast aufgebaut: Thomas hat in den USA viel Geld verdient, ist aber nicht so recht glücklich geworden und will nun in seinem Heimatdorf eine Frau finden. Der Berger-Karl ist bodenständig geblieben, verheiratet und Vater zweier Kinder. In seiner Freizeit bessert er eine für das Erzgebirge typische Pyramide aus, die sein Großvater geschnitzt hat. Das Arbeiten an der Pyramide hat ihn mit seinem Schicksal versöhnt, denn die Pyramide, so der Bergmann, »das ist mein Amerika. Immer, wenn mir das Leben zu sehr auf den Leib rückte, riß ich nicht aus, sondern schnitzte so ein Figürchen und arbeitete mich daran gesund. Und nun macht es allen Freude. $\aleph^{36}$ Beide Charaktere zeigen Tatkraft: Doch während Thomas in die USA geht, lenkt der Berger-Karl seine Tatkraft kreativ in traditionelles Kunsthandwerk um. Verankert in der regionalen wie in der Familientradition findet er die Zufriedenheit, die der Auswanderer nun zuhause sucht. Schließlich erleuchtet das Strahlen der Kerzen auf der Weihnachtspyramide das bescheidene Glück; der zurückgekehrte Auswanderer plant, seine Jugendliebe Lotte zu heiraten - die durch seine Abwesenheit gestörte Idylle ist wieder in Ordnung.

Die Tugend des Sich-in-die-Gegebenheiten-Einrichtens war immer wieder Thema in der Sendereihe. Die folgende Charakterisierung des Landboten zeigt, wie diese Tugend bewertet wurde: Der Landbote sei »kein weltfremder Alter [...], kein Flüchtling aus den Forderungen des Tages, sondern ein guter Geist, der allen

33 | Günter Häntzschel: »Idylle«, in: Harald Fricke (Hg.), Reallexikon der deutschen Literaturwissenschaft. Neubearbeitung des Reallexikons der deutschen Literaturgeschichte, 3. Aufl., Berlin, New York: W. de Gruyter 2000, S. 122 f.

34 | Detlev Peukert: Volksgenossen und Gemeinschaftsfremde. Anpassung, Ausmerze und Aufbegehren unter dem Nationalsozialismus, Köln: Bund Verlag 1992.

35 | Die Folge »Deutscher Kalender: Dezember 1937. Ein Monatsbild vom Königswusterhäuser Landboten« ist abgedruckt in: Karl Karst (Hg.): Günter Eich. Die Hörspiele. Gesammelte Werke in vier Bänden, Bd. II, Frankfurt/Main: Suhrkamp, S. 71-101.

36 | »Deutscher Kalender: Dezember 1937«, abgedruckt in: K. Karst: Günter Eich, S. 100 . 
Notwendigkeiten Sinn verleiht «. ${ }^{37}$ Diese Notwendigkeiten wurden zunehmend auf Grundlage der Anforderungen des nationalsozialistischen Staates formuliert. Vor allem die Landflucht und die moderne Großstadt stellten die implizite Folie, auf der die Vorteile eines ruhigen Lebens in überschaubarer Gemeinschaft geschildert wurden. Zusammenwirkend mit dem Schauplatz Dorf wurden hier eine Ganzheit und ein einfaches Leben vorgeführt, in der sich mit etwas gutem Willen alle Probleme lösen lassen. ${ }^{38}$

Im überlieferten Material finden sich noch weitere Heimkehrergeschichten; in zweien davon lockten die Versuchungen der Großstadt. ${ }^{39}$ Wie in der Dezemberfolge wird auch im Januar 1937 ein zurückgekehrter Sohn freudig wieder aufgenommen. Dies erinnert an das biblische Motiv des verlorenen Sohnes, der hier allerdings nicht heim zu seinem Vater oder heim zu Gott findet, sondern in die »Volksgemeinschaft « heimkehrt. ${ }^{40}$ Eine Abwandlung dieses Motivs findet sich in der Folge vom Oktober 1938. In dieser Folge entscheidet sich ein Matrose gegen das Landleben, obwohl es ihm gefällt und er sich in ein Mädchen aus dem Dorf verliebt hat. Auch hier wird verdeutlicht, dass jeder einen festen Platz hat, an den er gehört und an dem er bleiben sollte. ${ }^{41}$

Die >Morak der Geschichten wurde durch die zu Beginn einer jeden Folge zitierten Reaktionen der Hörer/-innen noch einmal verstärkt. So bedankte sich z. B. zum sechsjährigen Jubiläum ein Hörer dafür, dass ihn der Landbote für den Landdienst geworben habe; eine Hörerin berichtete, dass sie sich mit dem Leben in ihrer »kleinen, stickigen Welt« abgefunden habe, da ihr die Sendung zeige, dass sich auch im Kleinen die große Welt wiederfinden lasse, und anderes mehr. ${ }^{42}$

Die Kalendergeschichte, die stets eine didaktische Absicht hat, zeigte sich hier im Konzept der NS-Volksgemeinschaft verortet. Sie führte vor, wie Charaktere den Wert des Sich-Bescheidens an dem jeweils zugewiesenen Platz lernen sollten. Wem dies gelang, der erhielt seine Gratifikation im `Sein ‘. Allerdings mussten dies anscheinend überwiegend Männer lernen; Weiblichkeit wurde gern so präsentiert, als sei ihr diese Eigenschaft immanent.

37 | »Der Königswusterhäuser Landbote«, Beitrag aus Anlass des vierjährigen Jubiläums von M. Raschke und G. Eich, in: BA Koblenz, R 78, Nr. 831, Pressedienst DS (Oktober 1937), S. $8 \mathrm{f}$.

38 | Gemeint sind z. B. Probleme wie das der Müllerstochter, deren Vater nicht erlaubt, dass sie einen Bauernsohn heiratet, oder das der alten Magd, die auf dem Hof, auf dem sie ihr Leben lang gearbeitet hat, nicht bleiben darf, vgl. SLUB Dresden, App. 2521, Ms.-Nr. 2028, S. 37.

39 | Vgl. H.-U. Wagner: »Radiographie«, a. a. O., S. 173 f.

40 | Vgl. dazu ausführlich H.-U. Wagner: »Karriere ohne Kompromiss«, a. a. O., S. 9-11, Raschke hat dieses Motiv mehrfach verarbeitet (ebd.).

41 | Vgl. H.-U. Wagner: »Radiographie«, a. a. O., S. 193.

42 | Vgl. SLUB Dresden, App. 2531, Ms.-Nr. 2047, S. 1 ff., Verfasser dieser Folge ist vermutlich Martin Raschke. 


\section{Weibliches Sein}

In den ersten zwei Jahren der Ausstrahlung des »Königswusterhäuser Landboten« (1933-1935) fand eine Gleichsetzung von Frauen mit Natur und Fruchtbarkeit statt, die über weibliche Sagengestalten hergestellt wurde, etwa den Spukgeist Gret und die Kornmutter. ${ }^{43}$ Bei der Gret handelt es sich um einen der Geister, die in der Zeit zwischen den Jahren, an Weihnachten und Dreikönige - der Spukzeit -, umherziehen. Gret kann den Menschen Vergangenheit und Zukunft zeigen. Sie wird vom wilden Jäger verfolgt und findet Schutz in der Kammer des schlafenden Landboten. ${ }^{44}$ Als der Landbote erwacht, ist die wilde Jagd gerade akustisch vorbeigezogen - laut Regieanweisung haben Wind, Pferdetraben und Hundebellen nachgelassen. Der Landbote folgt Grets Aufforderung, ihr in die Augen zu schauen, und sieht dort das alte und das neue Jahr: »Aus der Vergangenheit kommen jetzt die Väter in langsamem Zuge [...] und aus der Zukunft die ungeborenen Enkel. Schatten und erst Kommende reichen sich die Hände, damit die Brücke gebaut sei, die Brücke von Jahr zu Jahr. « ${ }^{45}$ Dies ist nicht nur eine Brücke zwischen den Jahren. Der Geist Gret stellt das Bindeglied zwischen der Vergangenheit und der Zukunft, zwischen Vätern und Söhnen dar. Gret kann Vergangenes und Zukünftiges nicht beeinflussen, aber sichtbar machen und verbinden. ${ }^{46}$

Ähnlich naturhaft seiend und keinem Alltag unterworfen wird die Kornmutter dargestellt. Die Tochter des Bauern, Betty, die den Schnittern das Mittagessen bringen soll, begegnet ihr auf dem abgeernteten Feld. Die Kornmutter ist eine goldblonde, blauäugige Frau. Ihre Tätigkeit scheint nur darin zu bestehen, in den Himmel zu schauen und sich das Haar vom Wind kämmen zu lassen. Es handelt sich um einen der Korngeister bzw. -dämonen, die die Fruchtbarkeit des Landes beeinflussen kön-

43 | Hörfolge Oktober 1935 (SLUB Dresden, App. 2531, Ms.-Nr. 2028): Hier werden zum zweijährigen Bestehen der Sendereihe einzelne Szenen aus den vorangegangenen Sendungen erneut verwertet; die Sendung vermittelt so einen Querschnitt durch die ersten beiden Jahre. Handlungsrahmen ist hier der Jahresablauf auf einem Bauernhof in einem kleinen Dorf.

44 | In den zwölf Nächten zwischen Weihnachten und Dreikönige sind laut Überlieferung zahllose Geister und Seelen unterwegs. Eine der überregional bekanntesten Erscheinungen ist die wilde Jagd: Je wilder sie ist, desto fruchtbarer wird das Jahr. Vgl. Verband deutscher Vereine für Volkskunde (Hg.): Handwörterbuch des deutschen Aberglauben, Abt. I: Aberglaube, Bd. 9, Berlin 1938/41, Sp. 981 ff. Ein mit dem wilden Jäger verbundenes Motiv, auf das Eich hier zurückgreift, ist die so genannte Frauenjagdsage. Grundzüge dieser Sage sind: Ein weibliches, oft dämonisches Wesen wird vom wilden Jäger verfolgt; ein menschlicher Beobachter kann die Verfolgte beschützen oder schaut ihren Tod mit an und erhält einen Teil der Beute, vgl. Leander Petzold: Kleines Lexikon der Dämonen und Elementargeister, München: C. H. Beck 1990, S. $188 \mathrm{ff}$.

45 | SLUB Dresden, App. 2531, Ms.-Nr. 2028, S. 3.

46 | Diese Sagengestalt haben die Autoren im Gegensatz zu anderen sehr stark ausgeschmückt: Die Eigenschaft, den Menschen Vergangenheit und Zukunft zu zeigen, sowie der Name Gret sind nicht ursprünglich Bestandteil der »Frauenjagdsage«, vgl. L. Petzold: Dämonen, S. $188 \mathrm{ff}$. 
nen und von deren guten Willen die Landbevölkerung abhängig ist. ${ }^{47}$ Die Gestalt der Kornmutter scheint die Phantasie der Menschen angesprochen zu haben, denn noch zum vierjährigen Jubiläum 1937 wird sie vom Landboten erneut beschrieben als »Mutter der Felder, [...] [ein] Geist der Fruchtbarkeit [...], ein Wesen von Fleisch und Blut, das über den Saaten schwebt und seine Wohnung im Schatten der Ähren hat ${ }^{48}$

Sowohl bei Gret als auch der Kornmutter wird die fruchtbare und lebensspendende Seite von Weiblichkeit in den Vordergrund gestellt. Das Bedrohliche der Geister bzw. Dämonen, die hier als Vorlage dienten, tritt nur noch in Form von Ehrfurcht hervor. Die Natur erscheint in diesen weiblichen Sagengestalten gezähmt und freundlich und macht das Idyllische der Serie noch einmal deutlich. Durch den Wegfall des bedrohlichen Aspekts wird Weiblichkeit gleichzeitig auf fruchtbare Passivität und die Funktion festgelegt, die Generationen der Enkel und Väter zu verbinden.

Diese Passivität charakterisiert auch die Repräsentation weiblicher Figuren in der Weihnachtshörfolge von 1937, in der Thomas aus den USA in sein Dorf im Erzgebirge heimkehrt. In der oben kursorisch vorgestellten Folge bestimmt der Kontrast zweier männlicher Charaktere die Handlung; weibliche Charaktere stehen eher im Hintergrund; sie klöppeln, ziehen Kinder groß und warten, z. B. auf die Heimkehr des Sohnes oder der Jugendliebe. Passivität ist hier positiv besetzt, denn das Warten wird belohnt: Die in ihrer Heimat verankerte Lotte, die nicht mit nach Amerika möchte, gewinnt letztlich doch die Zuneigung ihrer Jugendliebe Thomas.

\section{Anpassung der Geschlechterordnung an die wechselnden Anforderungen der 1930er Jahre}

Zum vierjährigen Jubiläum wiederholte der Deutschlandsender einzelne der bislang ausgestrahlten Episoden. Dazu gehörte auch die Ansprache des Landboten zum Erntedankfest, an dem zugleich ein neugeborenes Mädchen getauft wurde. Mit diesem zeitlichen Zusammenfall wurde erneut eine Verbindung zwischen der Fruchtbarkeit der Felder und weiblicher Fruchtbarkeit hergestellt. Die Geburt einer Tochter anstelle eines Sohnes, die nicht auf ungeteilte Freude stößt, wird zum Anlass genommen, nachdrücklich auf die Bedeutung von Müttern für den einzelnen Mann und für die Gesellschaft hinzuweisen:

»[...] [Ihr] bedenkt nicht, daß es eine Mutter ist, die in dem Kinde uns allen heranwächst, eine Mutter, von der viele Söhne und viele Töchter zu ihrer Zeit ausgehen werden, eine Mutter, in der ein gutes Teil unserer Zukunft beschlossen ist. « ${ }^{49}$

47 Halme oder eine Garbe sollten den Geist an das Feld binden und dem Willen der Bauern unterwerfen, vgl. Wörterbuch der deutschen Volkskunde, neu bearb. von Richard Beitl unter Mitarbeit von Klaus Beitl, 3. Aufl., Stuttgart: Kröner 1974, S. 470.

48 | BA Koblenz, R 78, Nr. 831, Pressedienst DS (Oktober 1937), S. 5.

49 | Ebd., Hervorhebung d. V.; diese Episode ist gekürzt veröffentlicht in: Martin Raschke/Günter Eich: »Aus dem deutschen Kalender«, in: Die Literatur 41 (1938/39), S. 549 f. 
Mädchen und Frauen werden dabei nicht nur auf die Gebärfunktion beschränkt, sondern ihre Arbeit in der Familie wird auf durchaus individueller Basis aufgewertet. Die Männer werden zur Dankbarkeit und Wertschätzung ihrer Mutter angehalten:

»[...] wollt ihr denn [...] die Millionen Stunden vergessen, die Mütter mit wunden Augen am Bett ihrer Söhne wachten, um sie uns zu erhalten? [...] Mancher säße auch wohl nicht in Ehren unter uns, wenn nicht seine Mutter mit stetem Mahnen ihm den rechten Weg gewiesen hätte. $\aleph^{50}$

Die Aufwertung von Mädchen und Frauen wurde an die erzieherischen Leistungen gebunden, die Mütter für die Gesellschaft erbracht hatten. In dieser vom Landboten zur Taufe des neugeborenen Mädchens gehaltenen Ansprache klang an, dass Mütter ihre Kinder der Gemeinschaft zur Verfügung stellen sollten. Der Landbote formulierte den Anspruch, den die »Volksgemeinschaft» auf Kinder erhob, noch deutlicher: „Söhne, wer liebt sie nicht! [...] und wer ist nicht stolz, sie zum Schutze unserer Heimat mit ihrem Blut bereit zu sehen? « ${ }^{51}$

Hier wurde eine Ideologie von Opfer und Verzicht deutlich, die sich im Rahmen der Kriegsvorbereitungen leicht auf das männlich gedachte Vaterland übertragen ließ. Denn Mütter und Ehefrauen sollten im Kriegsfall die Einziehung ihrer männlichen Angehörigen nicht nur dulden, sondern sie auch ermutigen. Aus der Ferne sollten sie ihre Söhne, Ehemänner und Väter durch aufmunternde Briefe aufbauen. Diese Handlungsanweisung an Frauen wurde in der Hörfolge zum sechsjährigen Jubiläum im Oktober 1939 klipp und klar formuliert. ${ }^{52}$ In dieser Hörfolge arrangiert sich eine junge Frau, Käthe, damit, dass ihr Verlobter Erich eingezogen wurde und sie ihn vielleicht verliert. Käthe wird mit ihrer Mutter kontrastiert, einer fest im Leben stehenden Bäuerin, die der Mittelpunkt des Haushaltes ist und alles organisiert. Die Verhaltensvorschriften für junge Frauen in Käthes Situation werden von ihrer Mutter formuliert. So zerreißt sie die erste Variante von Käthes Brief an Erich, der ihr zu weinerlich ist, und diktiert ihr einen kurzen, aufbauenden Brief. ${ }^{53}$ Als Erich zu Besuch kommt, empfiehlt sie ihrer Tochter, ihm entgegenzurennen und ihn »mit einem Schmatz« zu begrüßen. ${ }^{54}$

50 | BA Koblenz, R 78, Nr. 831, Pressedienst DS (Oktober 1937), S. 6.

51 I Ebd. Hier schwingt bereits die Vorbereitung auf den Krieg mit.

52 | Dabei handelt es sich nicht um eine spezifisch nationalsozialistische Funktionalisierung bürgerlicher Weiblichkeitsideologie, vgl. Angelika Tramitz: »Vom Umgang mit Helden. Kriegs(vor)schriften und Benimmregeln für deutsche Frauen im Ersten Weltkrieg«, in: Peter Knoch (Hg.), Kriegsalltag. Die Rekonstruktion des Kriegsalltags als Aufgabe der historischen Forschung und der Friedenserziehung, Stuttgart: Metzler 1989, S. 84-113.

53 | SLUB Dresden, App. 2521, Ms.-Nr. 2047, S. 4 f. Verlangt wurde, dass Briefe an die Front das Positive betonten, um die Soldaten aufzumuntern, vgl. Ute Benz: Frauen im Nationalsozialismus. Dokumente und Zeugnisse, München: C. H. Beck 1993, S. 36.

54 | SLUB Dresden, App. 2521, Ms.-Nr. 2047, S. 20 f. 
In einem Gespräch zwischen Käthe und dem Landboten über Vergänglichkeit und Ewigkeit weist der Landbote sie auf die »Ewigkeit« hin, die sie in sich trägt, mit anderen Worten auf ihre zukünftigen Kinder. ${ }^{55}$ Dem schließt sich ein Gedicht über die Nornen, die germanischen Schicksalsgöttinnen, an. ${ }^{56}$ Durch das Gedicht wird die Aussage des Landboten unterstützt, der Käthes Angst vor Erichs Tod mit dem Hinweis auf seine Einbindung in eine Gemeinschaft beschwichtigt, die trotz des möglichen Todes eines Einzelnen erhalten bleibt:

»Wer allein ist unterm Himmel, den fällt leicht ein tückischer Wind an und verweht ihn und jede Kugel trifft ihn tödlich. Den aber, den tausend Bänder binden, Frau und Kinder, Väter und Mütter, Haus und Feld, der steht immer wie hinter einem Schilde. $\aleph^{57}$

Die Heimat wird hier als \Schild `des Soldaten beschrieben, der allerdings gleichzeitig die Heimat schützt. Denn anschließend wird durch die Erwähnung des Sternbildes Orion, der als »Wächter [...] mit dem Schwerte vor der Götterburg unserer Väter stand «, ${ }^{58}$ der Soldat als Beschützer charakterisiert. Die `Schild`-Funktion der Heimat besteht darin, dem Soldaten einen Grund für den Kampf zu geben - er weiß, wofür er kämpfen soll. Wehrlose Weiblichkeit, die es im Interesse eines Fortbestehens des >Volkes` zu beschützen gilt, steht hier im Endeffekt für >Heimat<. Durch die Schutzlosigkeit der weiblich gedachten Heimat erscheinen gewalttätige Handlungen gerechtfertigt. Damit die Illusion der Rechtmäßigkeit von Aggression erhalten bleibt, müssen diese Handlungen von Seiten der Schutzbedürftigen gebilligt werden. ${ }^{59}$ Die Schutzlosigkeit der Frauen wird in den Monatsbildern des $\gg$ Königswusterhäuser Landboten« konstruiert, indem die ebenfalls dargestellte weibliche Stärke und Macht auf den Bereich der Fruchtbarkeit und des Häuslichen begrenzt wird. Deswegen müssen die weiblichen mythischen Gestalten auch ihres bedrohlichen Aspektes beraubt werden.

Die Konstruktion wehrloser Weiblichkeit schon zu >Friedens $\triangleleft-Z e i t e n$ als etwas, das es zu schützen gilt bzw. für das die Zukunft geplant und gestaltet wird, ist essentiell für das männliche Selbstverständnis als Beschützer und wurde im »Königs-

55 | SLUB Dresden, App. 2521, Ms.-Nr. 2047, S. 8 f.

56 | Hier kommt die Angst vor dem Tod zur Sprache, aber die Nornen beruhigen den Beobachter: »Doch die dunklen Frauen weben/und sie winken mit der Hand/mir, den Bangnis lähmend band/aus der Tiefe neues Leben« (SLUB Dresden, App. 2521, Ms.-Nr. 2047, S. 10). Bei den Nornen handelt es sich um die drei Schicksalsgöttinnen der germanischen Mythologie, die als Weberinnen dargestellt werden, da sie die Lebensfäden aller Lebewesen in der Hand haben, das Leben Einzelner also auch beenden können.

57 I SLUB Dresden, App. 2521, Ms.-Nr. 2047, S. 15.

58 | SLUB Dresden, App. 2521, Ms.-Nr. 2047, S. 15.

59 | Vgl. Judith Hicks Stiehm: »The Protected, the Protector and the Defender «, in: Women's Studies International Forum 5 (1982), Heft 3/4, S. 367-376, hier S. 370 ff. Wie erfolgreich diese Mechanismen im Nationalsozialismus waren, wird beispielhaft aufgezeigt von: Christina Thürmer-Rohr: »Liebe und Lüge: `Meine geliebten Kinderchen!««, in: Dies., Vagabundinnen. Feministische Essays, Berlin: Orlanda Frauenverlag 1987, S. 57-75. 
wusterhäuser Landboten« prototypisch dargestellt. Das Konstrukt des soldatischen Mannes wird in dieser Folge nur im Gespräch zwischen Käthe und ihrer Mutter bzw. dem Landboten thematisiert (anders z. B. in der Folge vom April 194060). Im Mittelpunkt der Erzählung steht mit Käthe ein weiblicher Charakter, der sich zu Beginn des Krieges mit neuen `Gegebenheiten (hier der Einberufung) abfinden muss - erneut wird hier der Appell deutlich, die Umstände nicht zu hinterfragen, sondern zu akzeptieren, wie sie sind.

\section{Erzählstruktur}

Ein Kennzeichen der Gattung war die Personifizierung des Kalenders in einem »Kalendermann«, der sich als Dialogpartner des Lesers bzw. der Leserin anbot und die Moral der Geschichte erläuterte. Der Dialog und die direkte Ansprache von Leserin und Leser wurden damit zur typischen Erzählweise schon des frühen, nicht literarisierten Volkskalenders. ${ }^{61}$ Mit der Wahl der Gattung war also bereits die Entscheidung für eine Struktur gefallen, die eine zentrale männliche Erzählinstanz vorsah, ohne die die Erzählung nicht stattfinden kann. ${ }^{62}$ Damit ist auch die Erzählerstimme festgelegt, die von ihrer Textposition her die erwünschte Lesart der Geschichten bestimmt. Denn:

»[...] mit unterschiedlichen Erzählerstimmen [...] erleben [die Rezipientinnen und Rezipienten] die fiktive Welt und sind eingeladen, die von dieser Stimme geäußerten Vermutungen, Urteile und Werte zu teilen oder aber auch dieser Stimme zu misstrauen. [Erzähl-]Stimmen konstituieren Textsubjekte und damit Subjektpositionen, die geschlechtlich markiert sein können $[\ldots] . \ll^{63}$

Da der Landbote als Textsubjekt mit hoher Autorität konstruiert wird, zielen die Autoren darauf ab, ihm und damit auch seinen Werturteilen Glaubwürdigkeit zu verleihen.

Die Autorität des Landboten entsteht $u$. a. dadurch, dass er als Repräsentant des

60 | Vgl. H.-U. Wagner: »Radiographie«, a. a. O., S. 207.

61 | Vgl. Jan Knopf: Die deutsche Kalendergeschichte. Ein Arbeitsbuch. Frankfurt/Main: Suhrkamp 1973, S. 23, sowie J. Knopf: »Kalendergeschichte«, a. a. O., S. 218.

62 | Diese narrative Struktur bot sich für eine Umsetzung im Rundfunk geradezu an: Auch die vom Reichssender Köln im Landfunk ab 1936 ausgestrahlte Reihe »Der Kiepenkerl packt aus. Guter Rat für Stadt und Land« basierte auf einer zentralen Kalendergestalt, die als Dialogpartner für die Rezipientinnen und Rezipienten fungieren sollte. Mit einem so genannten »wöchentlichen Arbeitskalender« kam sie traditionellen Volkskalendern sehr viel näher als der »Königswusterhäuser Landbote«. Vgl. Daniela Münkel: »Radio für das Land. Der Landfunk in der NS-Zeit«, in: Westfälische Forschungen 47 (1997), S. 427-451, hier S. 446 f., zu traditionellen Volkskalendern vgl. J. Knopf, Deutsche Kalendergeschichte, S. 14 ff.

63 | Birgit Wagner: »Erzählstimmen und mediale Stimmen. Mit einer Analyse von Assia Djebars Erzählung Die Frauen von Algier«, in: Nieberle/Strowick (Hg.), Narration und Geschlecht (2006), S. 141-158, hier S. 143 f. 
Rundfunks in bestimmte Landstriche reist und dort Bräuche und Ereignisse thematisiert. Somit entscheidet er über den Gegenstand des medialen Erzählens. Dies demonstriert seine Handlungsfähigkeit, denn die Figur ist in der Sendereihe so verortet, dass sein Erscheinen die Aufmerksamkeit auf das ausgewählte Geschehen lenkt und deren Wiedergabe in der jeweiligen Folge veranlasst. Bei ihm liegt die »diskursive Autorität «. ${ }^{64}$ Gleichzeitig sind die Kalenderbilder durch die Charakteristika seriellen Erzählens geprägt. Die narrative Struktur jeder Folge in der Sendereihe ist in sich abgeschlossen, die Grundsituation und die Hauptcharaktere bleiben gleich. Der Vorteil von Serien für den Rundfunk ist, dass sie »über Monate und Jahre hinweg Erzählungen anbieten und [Rezipientinnen und Rezipienten] [...] binden « können. ${ }^{65}$ Der serielle Charakter der Sendereihe stärkt den Status der Kalendergestalt: AuBer dem Landboten kehren nur wenige Charaktere wieder (wie z. B. der ebenfalls umherziehende Landbriefträger Apfelstädt ${ }^{66}$ ), so dass nur er über die Jahre zur vertrauten Gestalt für die Hörenden wird. Dies ermöglicht einen emotionalen Bezug zum Hauptcharakter der Sendung, der vermutlich auch zum Erfolg der Sendereihe beigetragen hat. Die Personifizierung der Kalendergestalt - bereits in der Gattung angelegt - wird so weit geführt, dass der Regisseur der Serie, Helmuth Hansen, 1937 als Landbote kostümiert auf der Rundfunkausstellung erscheint. ${ }^{67}$

Die Figur des Landboten gehört anscheinend gleichzeitig zu den für Raschke, den anderen Autor der Sendefolgen, typischen »kommentierenden und wertenden, manchmal >omnipotent` erscheinenden Erzählerfiguren«. ${ }^{68}$ Der Landbote nimmt kaum an der Handlung teil und seine Lebensgeschichte oder -umstände bleiben weitgehend im Dunkeln. Seine Funktion ist die des Bezeugens, Kommentierens und Wertens. Allerdings greift er immer wieder ein, um einen Konflikt zu schlichten oder einem jungen Liebespaar zu seinem Glück zu verhelfen. Sowohl als Beobachter und Berichterstatter wie Schlichter konstituiert der Landbote die Sendereihe. Diese männliche Figur mit Erzählfunktionen liefert wertgebundene Interpretationen und hat so die Definitionsmacht über das Geschehen. Durch den Landboten wird die bevorzugte Lesart, die hegemoniale Bedeutung zugewiesen. Damit entsteht auch eine hierarchische Struktur: Die anderen Charaktere in den Monatsbildern sind in Bezug auf Glaubwürdigkeit und Autorität der Erzählinstanz Landbote untergeordnet. In Bezug auf die Konstruktion von Weiblichkeit bedeutet

$64 \mid$ Ebd., S. 143.

65 | Vgl. Knut Hickethier: Die Fernsehserie und das Serielle des Fernsehens (Kultur Medien - Kommunikation: Lüneburger Beiträge zur Kulturwissenschaft, 2), Lüneburg: Universität 1991, S. 8.; Knut Hickethier: Film- und Fernsehanalyse (Sammlung Metzler, 277), Stuttgart, Weimar: Metzler 1996, S. 204.

66 | Apfelstädt taucht z. B. in den Folgen 12/1936 und 10/1937 auf, vgl. H.-U. Wagner: »Radiographie«, a. a. O., S. 172 und 183.

67 | Sondersendung »Der Königswusterhäuser Landbote auf der Rundfunkausstellung«, 30.7.1937, vgl. H.-U. Wagner: »Radiographie«, a. a. O., S. 177 f.

$68 \mid$ Wilhelm Haefs: »)Die götterlose Zeit will enden ... <. Martin Raschke als Erzähler im >Dritten Reich««, in: Ders./Walter Schmitz (Hg.), Martin Raschke (1905-1943): Leben und Werk, Bamberg: Thelem 2002, S. 79-106, hier S. 81. 
dies zunächst, dass weibliche Charaktere keine Stimme haben. Eine dem Landboten vergleichbare weibliche Erzählinstanz gibt es nicht. Für männliche und weibliche Figuren gilt, dass die Anwesenheit des Landboten sie sichtbar macht. Szenen, an denen der Landbote nicht teilhat, sind darüber hinaus durch die Erzählperspektive eines allwissenden, auktorialen Erzählers geprägt, der ebenfalls männlich gedacht ist. Dies zeigt:

»[...] erzählendes Geschlecht und erzähltes Geschlecht [stehen] in einem engen korrelativen Wechselverhältnis, weil die jeweilige Besetzung der Erzählpositionen maßgeblichen Einfluss darauf hat, welche Vorstellungen von >Weiblichkeit` und `Männlichkeit` in einem Text entworfen werden. ${ }^{69}$

Augenfällig ist, dass die Vorstellungen von Männlichkeit nach Alter hierarchisiert sind. Die Weisheit des Alters gestattet dem Landboten sein begütigendes, Erfolg und Frieden stiftendes Eingreifen. Jüngere männliche Charaktere werden im Prozess der Einpassung in die Stelle der Gemeinschaft präsentiert, die Beruf oder Herkunft ihnen offenlassen - ein kleinbürgerliches Modell, das nichtsdestotrotz zeitgenössische Erfahrungshorizonte einbezieht. Dabei weist ihnen der Landbote den Weg, hier z. B. in der Folge vom Dezember 1937:

»Es gibt, glaube ich, Herr Thomas, zweierlei Menschen [...]. Solche Menschen, die unablässig ihren Traum auf der Erde verwirklicht suchen, und solche, die sich mühen, daß der Ort, an den sie vom Schicksal gestellt sind, dem schönsten Menschtraum von der Erde ähnlicher und ähnlicher wird, ganz unbekümmert darum, was ringsum sie lockt. $\ll^{70}$

Dieses Sich-Anpassen an die Gegebenheiten erinnert auch an die Anforderung an Soldaten, an dem Ort ihre Aufgaben zu erfüllen, an den sie gestellt werden. Amberger hat in der Analyse von Kriegsromanen des Ersten Weltkrieges gezeigt, dass das Prinzip militärischen Gehorsams nicht als Unterwerfung und Einordnung, sondern als »positives Aufgehen in einer sinnstiftenden Gemeinschaft dargestellt « wird. ${ }^{71}$ Dieses Prinzip zeigt sich hier auch in der medialen Konstruktion der »Volksgemeinschaft« als sinnstiftender Gemeinschaft.

$69 \mid$ Ebd.

70 | »Deutscher Kalender: Dezember 1937«, abgedruckt in: K. Karst, Günter Eich, S. 94.

71 | Vgl. Waltraud Amberger: Männer, Krieger, Abenteurer. Der Entwurf des »soldatischen Mannes« in den Kriegsromanen über den Ersten und Zweiten Weltkrieg (Frankfurter Beiträge zur neueren deutschen Literaturgeschichte, 2), Frankfurt/Main: R. G. Fischer 1984, S. 66. 


\section{Gesellschaftlicher Kontext der medialen Repräsentationen}

Die Sendereihe überstand die Programmumstrukturierungen der ersten Jahre der nationalsozialistischen Herrschaft unbeschadet, war also für ihr Publikum weiter von Interesse. Die Attraktivität der Sendung beruhte darauf, dass sie der zunehmenden Verstädterung und der damit einhergehenden Beschleunigung und Zerrissenheit gesellschaftlichen Lebens in der Moderne ein Gegenbild zur Verfügung stellte: ein einfaches ländliches Leben mit idyllischen Zügen, das im Laufe der 1930er Jahre immer stärker Bezug auf zeitgenössische Themen nahm, die allerdings auch immer öfter von der NS-Propaganda vorgegeben wurden. Hier wurde Zukunftsangst durch die Beschwörung >ewiger Werte beschwichtigt. $^{72}$ Die Sendereihe vereinte das Werben für innere Werte wie Kreativität und ländlich Beschauliches mit der Aufforderung, sich einzurichten, dem Abfinden mit den Gegebenheiten. Die Erzählstruktur unterstützte diese Botschaft, denn neben dem entsprechend angelegten Geschehen warb auch die diskursive Autorität der Reihe, der Landbote, dafür.

Die Hierarchie der kontrastierenden Geschlechtskonstruktionen war in die Sendung eingebaut: Der Landbote war der narrative Dreh- und Angelpunkt der Sendung, er interpretierte und kommentierte das Geschehen und war damit auch der Figur des jungen Mannes übergeordnet, der immer wieder als Handelnder dargestellt wurde. Die der Natur verhafteten Frauen handelten hingegen selten. Das seltene Handeln von Frauen diente zudem lediglich der Unterstützung und Bestärkung männlichen Handelns.

Die Vorstellung von Gemeinschaft, in der Männer und Frauen entgegengesetzte Rollen übernehmen, kann nicht allein auf die Werte der Autoren oder der Redaktion, die stark in die Gestaltung der Folgen eingriff, zurückgeführt werden. Sie ist in einen breiten zeitgenössischen Diskurs einzuordnen. Die hier aufgezeigten Mythen des Soldaten und der Mutter sind nicht spezifisch für den Nationalsozialismus, sondern spätestens seit dem Ersten Weltkrieg nachweisbar. Ebenso war die Geschlechterpolarität Bestandteil des seit dem 19. Jahrhunderts entwickelten, bürgerlichen Frauenbildes. ${ }^{73}$ Im Nationalsozialismus wurde sie vereinnahmt. Ein polar gedachtes Geschlechterverhältnis war eine der strukturellen Unterscheidungen in der »Volksgemeinschaft«, so schlug sie sich auch in dieser medialen Inszenierung

72 | Vgl. dazu Adelheid von Saldern: »Kulturelle Praxisformen im Dritten Reich. Die Geschichtswissenschaft vor neuen Aufgaben«, in: Hinrich Bergmeier/Günter Katzenberger (Hg.), Kulturaustreibung. Die Einflußnahme des Nationalsozialismus auf Kunst und Kultur in Niedersachsen, Hamburg: Dölling und Galitz 1995, S. 10-118, hier S. 11 f.

73 | Vgl. Karin Stiehl: „Auf der Suche nach Weiblichkeitsbildern im Nationalsozialismus«, in: Barbara Determann et al. (Hg.), Verdeckte Überlieferungen. Weiblichkeitsbilder zwischen Weimarer Republik, Nationalsozialismus und Fünfziger Jahren (Arnoldshainer Texte, 68), Frankfurt/Main: Haag + Herchen 1993, S. 27-39; J. H. Stiehm: »Protector«, a. a. O.; Ute Frevert: »Geschlecht - männlich/weiblich. Zur Geschichte der Begriffe (1730-1990)«, in: Dies., >Mann und Weib, und Weib und Mann`: Geschlechter-Differenzen in der Moderne, München: C. H. Beck 1995, S. 13-60. 
nieder. Dazu traten die regionale Verortung im Herkunftsort und die Verwurzelung von Charakteren in traditioneller Volkskultur. Durch die Darstellung eines idealen Funktionierens der »Volksgemeinschaft« wurde deutlich gemacht, dass die Einordnung in diese Gemeinschaft Identitätsbildung und Sinnstiftung nach sich zog. Verunsicherungen, wie sie mit sozialer Differenzierung in einer modernen Gesellschaft einhergehen, konnten dadurch angesprochen und einfache Lebensmodelle als eine Lösung präsentiert werden.

Die Abkehr von der >großen Welt` verwies die Hörenden auf ihr alltägliches Umfeld, die private Idylle und konnte so eine Gleichgültigkeit gegenüber politischen Geschehnissen fördern. Dies traf sich dann mit den Rezeptionsinteressen der Hörenden, wenn Wünsche nach Geborgenheit, Glück und einem guten Leben nur noch im privaten Rahmen realisierbar erschienen. Eine solche Schlussfolgerung legt der Rückzug ins Private nahe, der z. B. sowohl von Schäfer wie auch von Peukert analysiert wurde. ${ }^{74}$ Anhand solcher Medienangebote konnte man sich die Bestätigung holen, dass es zumindest im privaten Rahmen noch möglich war, trotz widriger Umstände glücklich zu sein. Eingebettet in ein festes soziales und vor allem überschaubares Gefüge war die erfolgreiche Zweierbeziehung zwischen Mann und Frau konstitutiv für das Glück im Kleinen. So kehrt, wie gezeigt wurde, ein Heimkehrer der Kalendergeschichte nicht nur in sein Dorf, sondern auch zu seiner Jugendliebe zurück. Hier werden Vorstellungen von Männlichkeit und Weiblichkeit erkennbar, die nicht spezifisch für den Nationalsozialismus waren, sondern auf breitem gesellschaftlichem Konsens beruhten.

Nach den Verunsicherungen in den 1920er Jahren, ${ }^{75} \mathrm{zu}$ denen das Aufbrechen traditioneller Ordnungsstrukturen vor allem in Bezug auf Geschlecht sowie die wirtschaftliche Krise Ende der 1920er Jahre entscheidend beitrugen, zeigte sich in der traditionell didaktisch orientierten Gattung Kalendergeschichte der 1930er Jahre die Neuinszenierung männlicher Überlegenheit, die hier als weiser Alter auftrat, dem - zumindest narrativ - die jüngeren bzw. nicht so weisen Männer untergeordnet waren. Die jüngeren männlichen Charaktere besaßen trotzdem Handlungsfähigkeit. Damit stellte die Sendereihe eine hohe Bandbreite an männlichen Identifikationsangeboten bereit. ${ }^{76}$ Weiblichkeit blieb narrativ untergeordnet, traditionell ihr zugeschriebene Tätigkeiten wurden gleichzeitig aufgewertet, was Kundrus als »ordnende Versöhnung der Geschlechter [anstelle] beängstigender Vielfalt« be-

74 | Vgl. Hans Dieter Schäfer: »Das gespaltene Bewußtsein. Über die Lebenswirklichkeit in Deutschland 1933-1945«, in: Ders., Das gespaltene Bewußtsein. Deutsche Kultur und Lebenswirklichkeit, München: Hanser 1981, S. 114-162; D. Peukert: Volksgenossen, S. 89 f.

75 | Zur Vereinnahmung Martin Raschkes im Nationalsozialismus und seiner Verarbeitung des »Krisengefühls« vgl. H.-U. Wagner: »Karriere ohne Kompromiss«, a. a. O., S. 10 f.

76 | Zur Re-Inszenierung männlicher Überlegenheit vgl. Birthe Kundrus: »Geschlechterkriege. Der Erste Weltkrieg und die Deutung der Geschlechterverhältnisse in der Weimarer Republik«, in: Karen Hagemann/Stefanie Schüler-Springorum (Hg.), Heimat-Front. Militär und Geschlechterverhältnisse im Zeitalter der Weltkriege. Frankfurt/Main, New York: Campus 2002, S. 171-187, hier S. 179 f. 
schreibt. ${ }^{77}$ Die beschriebene spezifische diskursive Deutung von Geschlecht befriedigte für einen Teil der Hörenden ein Bedürfnis nach zumindest symbolischer Ordnung der Welt.

\section{Literaturverzeichnis}

\section{Unveröffentlichte Quellen}

Bundesarchiv (BA), R 78, Nr. 831, Pressedienst Deutschlandsender (Oktober 1937) (Bestand heute im BA Berlin)

Deutsches Rundfunkarchiv Frankfurt/Main (DRA):

Pressemitteilungen Deutschlandsender

Mitteilungen der Reichsrundfunkgesellschaft (Mitt. RRG)

Sächsische Landesbibliothek/Staats- und Universitätsbibliothek Dresden (SLUB Dresden), Nachlass Martin Raschke, App. 2521, daraus:

Manuskript-Nr. 2072, »Weihnachtsreise des Königswusterhäuser Landboten« (Sondersendung Dezember 1934).

Manuskript-Nr. 2028, »Deutscher Kalender: Oktober 1935. Ein Monatsbild vom Königswusterhäuser Landboten«- Manuskript-Nr. 2047, »Deutscher Kalender: Oktober 1939. Ein Monatsbild vom Königswusterhäuser Landboten«.

\section{Gedruckte Quellen}

»Deutscher Kalender: Dezember 1937. Ein Monatsbild vom Königswusterhäuser Landboten«, abgedruckt in: Karl Karst (Hg.), Günter Eich: Die Hörspiele. Gesammelte Werke in vier Bänden, Bd. II, Frankfurt/Main: Suhrkamp, S. 71-101.

Hadamovsky, Eugen: Der Rundfunk im Dienste der Volksführung (Gestalten und Erscheinungen der politischen Publizistik, 1), Leipzig: Noske 1934, S. 20.

Raschke, Martin/Eich, Günter: »Aus dem deutschen Kalender«, in: Die Literatur 41 (1938/39), S. 549 f.

\section{Literatur}

Amberger, Waltraud: Männer, Krieger, Abenteurer. Der Entwurf des ısoldatischen Mannes in den Kriegsromanen über den Ersten und Zweiten Weltkrieg (Frankfurter Beiträge zur neueren deutschen Literaturgeschichte, 2), Frankfurt/Main: R. G. Fischer 1984.

Benz, Ute (Hg.): Frauen im Nationalsozialismus. Dokumente und Zeugnisse, München: C. H. Beck 1993.

Cuomo, Glenn R.: Career at the Cost of Compromise. Günter Eich's Life and Work in the Years 1933-1945, Amsterdam u. a.: Rodopi 1989.

77 | B. Kundrus: »Geschlechterkriege«, a. a. O., S. 179. 
Frevert, Ute: »Geschlecht - männlich/weiblich. Zur Geschichte der Begriffe (1730-1990)«, in: Dies., 〉Mann und Weib, und Weib und Mann`: GeschlechterDifferenzen in der Moderne, München: C. H. Beck 1995, S. 13-60.

Haefs, Wilhelm: »»Die götterlose Zeit will enden ...<. Martin Raschke als Erzähler im 〉Dritten Reich«", in: Ders./Walter Schmitz (Hg.), Martin Raschke (1905-1943): Leben und Werk, Bamberg: Thelem 2002, S. 79-106.

Häntzschel, Günter: »Idylle«, in: Harald Fricke (Hg.), Reallexikon der deutschen Literaturwissenschaft. Neubearbeitung des Reallexikons der deutschen Literaturgeschichte, 3. Aufl., Berlin, New York: W. de Gruyter 2000, S. 122 f.

Hickethier, Knut: Die Fernsehserie und das Serielle des Fernsehens (Kultur - Medien - Kommunikation: Lüneburger Beiträge zur Kulturwissenschaft, 2), Lüneburg: Universität 1991.

Ders.: Film- und Fernsehanalyse, Stuttgart, Weimar: Metzler 1996.

Knopf, Jan: Die deutsche Kalendergeschichte. Ein Arbeitsbuch, Frankfurt/Main: Suhrkamp 1973.

Ders.: »Kalendergeschichte«, in: Harald Fricke (Hg.), Reallexikon der deutschen Literaturwissenschaft. Neubearbeitung des Reallexikons der deutschen Literaturgeschichte, 3. Aufl., Berlin, New York: de Gruyter 2000, S. 217 f.

Kundrus, Birthe: »Geschlechterkriege. Der Erste Weltkrieg und die Deutung der Geschlechterverhältnisse in der Weimarer Republik«, in: Karen Hagemann/ Stefanie Schüler-Springorum (Hg.), Heimat-Front. Militär und Geschlechterverhältnisse im Zeitalter der Weltkriege, Frankfurt/Main, New York: Campus 2002, S. 171-187.

Marßolek, Inge/Saldern, Adelheid von (Hg.): Zuhören und Gehörtwerden 1. Radio im Nationalsozialismus zwischen Lenkung und Ablenkung, Tübingen: edition diskord 1998.

Merten, Klaus: Inhaltsanalyse. Eine Einführung in Theorie, Methode und Praxis, Opladen: Westdeutscher Verlag 1980.

Mühlen-Achs, Gitta/Schorb, Bernd: »Einleitung«, in: Dies. (Hg.), Geschlecht und Medien, München: KoPäd Verlag 1995, S. 7-11.

Münkel, Daniela: »Radio für das Land. Der Landfunk in der NS-Zeit«, in: Westfälische Forschungen 47 (1997), S. 427-451.

Dies.: »Die Entwicklung der Programmstruktur«, in: Marßolek/Saldern (Hg.), Zuhören und Gehörtwerden 1 (1998), S. 95-105.

Nieberle, Sigrid/Strowick, Elisabeth (Hg.): Narration und Geschlecht. Texte - Medien - Episteme, Köln, Weimar, Wien: Böhlau 2006.

Nünning, Ansgar/Nünning, Vera: »Making Gendered Selves: Analysekategorien und Forschungsperspektiven einer gender-orientierten Erzähltheorie und Erzähltextanalyse«, in: Nieberle/Strowick (Hg.), Narration und Geschlecht (2006), S. 23-44.

Pater, Monika: »Rundfunkangebote«, in: Marßolek/Saldern (Hg.), Zuhören und Gehörtwerden 1 (1998), S. 172-187.

Pausch, Holger A./Herzog, Marianne: »Vergessene Texte, Schrift und Sprache. Beobachtungen zur Günter-Eich-Kontroverse«, in: Wirkendes Wort 45 (1995), Heft 1, S. 133-150. 
Petzold, Leander: Kleines Lexikon der Dämonen und Elementargeister, München: C. H. Beck 1990.

Peukert, Detlev: Volksgenossen und Gemeinschaftsfremde. Anpassung, Ausmerze und Aufbegehren unter dem Nationalsozialismus, Köln: Bund Verlag 1992.

Saldern, Adelheid von: »Kulturelle Praxisformen im Dritten Reich. Die Geschichtswissenschaft vor neuen Aufgaben«, in: Hinrich Bergmeier/Günter Katzenberger (Hg.), Kulturaustreibung. Die Einflußnahme des Nationalsozialismus auf Kunst und Kultur in Niedersachsen, Hamburg: Dölling und Galitz 1995, S. 10-118.

Schäfer, Hans Dieter: »Das gespaltene Bewußtsein. Über die Lebenswirklichkeit in Deutschland 1933-1945«, in: Ders., Das gespaltene Bewußtsein. Deutsche Kultur und Lebenswirklichkeit, München: Hanser 1981, S. 114-162.

Schmidt, Uta: »Radioaneignung«, in: Marßolek/Saldern (Hg.), Zuhören und Gehörtwerden 1 (1998), S. 243-360.

Stiehl, Karin: »Auf der Suche nach Weiblichkeitsbildern im Nationalsozialismus«, in: Barbara Determann et al. (Hg.), Verdeckte Überlieferungen. Weiblichkeitsbilder zwischen Weimarer Republik, Nationalsozialismus und Fünfziger Jahren, Frankfurt/Main: Haag + Herchen 1993, S. 27-39.

Stiehm, Judith Hicks: »The Protected, the Protector and the Defender«, in: Women's Studies International Forum 5 (1982), Heft 3/4, S. 367-376.

Stritzke, Nadyne: »(Subversive) Narrative Performativität. Die Inszenierung von Geschlecht und Geschlechtsidentitäten aus Sicht einer gender-orientierten Narratologie«, in: Nieberle/Strowick (Hg.), Narration und Geschlecht (2006), S. 93-116.

Thürmer-Rohr, Christina: »Liebe und Lüge: >Meine geliebten Kinderchen!«, in: Dies., Vagabundinnen. Feministische Essays, Berlin: Orlanda Frauenverlag 1987, S. 57-75.

Tramitz, Angelika: »Vom Umgang mit Helden. Kriegs(vor)schriften und Benimmregeln für deutsche Frauen im Ersten Weltkrieg«, in: Peter Knoch (Hg.), Kriegsalltag. Die Rekonstruktion des Kriegsalltags als Aufgabe der historischen Forschung und der Friedenserziehung, Stuttgart: Metzler 1989, S. 84-113.

Verband deutscher Vereine für Volkskunde (Hg.): Handwörterbuch des deutschen Aberglauben, Abt. I: Aberglaube, Bd. 9, Berlin 1938/41, Sp. $981 \mathrm{ff}$.

Vieregg, Axel: Der eigenen Fehlbarkeit begegnet. Günter Eichs Realitäten 19331945, Eggingen: Edition Isele 1993.

Wagner, Birgit: »Erzählstimmen und mediale Stimmen. Mit einer Analyse von Assia Djebars Erzählung Die Frauen von Algier«, in: Nieberle/Strowick (Hg.), Narration und Geschlecht (2006), S. 141-158.

Wagner, Hans-Ulrich: »Essay«, in: Ders., Günter Eich und der Rundfunk. Essay und Dokumentation, Potsdam: Verlag für Berlin-Brandenburg 1999, S. 25-106.

Ders.: »Kommentierte Radiographie«, in: Ders., Günter Eich und der Rundfunk. Essay und Dokumentation, Potsdam: Verlag für Berlin-Brandenburg 1999, S. $107-376$.

Ders.: ")Der Weg in ein sinnhaftes, volkhaftes Leben<. Die Rundfunkarbeiten von Martin Raschke«, in: Wilhelm Haefs/Walter Schmitz (Hg.), Martin Raschke (1905-1943): Leben und Werk, Bamberg: Thelem 2002, S. 79-106. 
Ders.: »Eine Karriere ohne Kompromiss. Martin Raschke und der Rundfunk 1928-1940«, in: Rundfunk und Geschichte 28 (2002), Heft 1/2, S. 5-21.

Wessels, Wolfram: Hörspiele im Dritten Reich. Zur Institutionen-, Theorie- und Literaturgeschichte, Bonn: Bouvier 1985.

Wörterbuch der deutschen Volkskunde, neu bearb. von Richard Beitl unter Mitarbeit von Klaus Beitl, 3. Aufl., Stuttgart: Kröner 1974. 